\title{
Incorporating Index of Fuzziness and Adaptive Thresholding for Image Segmentation
}

\author{
Umi Salamah ${ }^{1}$, Riyanarto Sarno ${ }^{2}$, Agus Zainal Arifin ${ }^{3}$, Anto Satriyo Nugroho ${ }^{4}$, \\ Ismail Ekoprayitno Rozi ${ }^{5}$, Puji Budi Setia Asih ${ }^{6}$ \\ ${ }^{1,2,3}$ Department of Informatics, Institut Teknologi Sepuluh Nopember, Surabaya, Indonesia \\ ${ }^{1}$ Department of Informatics, Universitas Sebelas Maret, Surakarta, Indonesia \\ ${ }^{4}$ Center for Information and Communication Technology Agency for the Assessment and Application of Technology \\ (PTIK-BPPT), Indonesia \\ ${ }^{5,6}$ Eijkman Institute for Molecular Biology, Indonesia
}

\section{Article Info}

Article history:

Received Jan 8, 2018

Revised Jun 28, 2018

Accepted Jul 10, 2018

\section{Keyword:}

Adaptive thresholding

Bimodal

Index of fuzziness

Significant contrast

Very irregular histogram

\begin{abstract}
Binary Segmentation of an image played an important role in many image processing application. An image that was having no bimodal (or nearly) histogram accompanied by low-contrast was still a challenging segmentation problem to address. In this paper, we proposed a new segmentation strategy to images with very irregular histogram and had not significant contrast using index of fuzziness and adaptive thresholding. Index of fuzziness was used to determine the initial threshold, while adaptive thresholding was used to refine the coarse segmentation results. The used data were grayscale images from related papers previously. Moreover, the proposed method would be tested on the grayscale images of malaria parasite candidates from thickblood smear that had the same problem with this research. The experimental results showed that the proposed method achieved higher segmentation accuracy and lower estimation error than other methods. The method also effective proven to segment malaria parasite candidates from thickblood smears image.
\end{abstract}

Copyright () 2018 Institute of Advanced Engineering and Science. All rights reserved.

\section{Corresponding Author:}

Umi Salamah,

Department of Informatics, Institut Teknologi Sepuluh Nopember Surabaya,

Jl. Teknik Kimia, Gedung Teknik Informatika,

Kampus ITS Sukolilo, Surabaya, 60111, Indonesia.

Email: umi14@mhs.if.its.ac.id

\section{INTRODUCTION}

Image segmentation is a critical process in many image processing applications such as object detection, shape recognition, and optical character recognition [1]. Segmentation accuracy determines the success or failure of computerized analysis procedures [2]. Global Thresholding is a popular tool used in image segmentation [3], because it easy implemented. Optimal condition of image segmentation is obtained if there is a significant contrast between the object and the background. The condition is characterized by bimodal formation with a deep valley in image histogram. The valley that separates the two modalities is the right location of the threshold [2].

The absence of an obvious splitting valley is due to noise and poor illumination, so the segmentation process becomes not optimal. Some studies based on statistical properties of the image histogram are addressed to solve the problem. Between-class variance is suggested by Otsu [4] to segment optimally the object of the image. Inter-class variance and intra-class variance are utilized by Arifin and Asano [5] to threshold the image by performing hierarchal clustering analysis. However, these methods do not constantly function well on ill-defined images that are impaired by noise and irregularly illuminated. A Measure of fuzziness is offered some studies to overcome the problem. Huang and Wang [6] minimize the measure of 
fuzziness to threshold the image. Tizhoosh [7] propose ultra fuzziness which is a new fuzzy measure based on the fuzzy set type II. However, the technique does not constantly function for images with a very irregular histogram

This problem get over by Tobias and Seara [8], Lopes et al [2], and Pratamasunu [9] use fuzzy similarity measure between graylevel that is more compatible with object edges and ambiguity, and it can prevent in minimum finding problems. The similarity calculation process involves one of the fuzzy measures, i.e. index of fuzziness $(I o F)$ to determine the appropriate fuzzy region. The result is very satisfying. However, it does not always work on very irregular histogram images and has not significant contrast. The segmentation accuracy decrease compared segmented result using the only $I o F$. This result happens because sometimes finding the minimum of a function is trapped on local optimal. Therefore, a segmentation strategy that can handle images that have very irregular histograms and low-contrast is needed. In this paper, we propose a new segmentation strategy to images with very irregular histogram and have not significant contrast using $I o F$ and adaptive thresholding. The result will be implemented on candidate parasite segmentation that most of the images have the same problem with this study.

\section{RELATED WORK}

\subsection{Index of Fuzziness}

Index of fuzziness is one type of fuzzy measure. These terms describe the measures of the proximity of a grey tone image to its two-tone version and also ambiguity in a fuzzy set. The low value indicates the low ambiguity among the elements. Crossover point modification of will yield in a diversity of these parameters and so a set of minima is acquired appropriate to the optimum threshold levels [10]. The IoF is defined [11] as

$$
I(A)=\frac{2}{n^{k}} d(A, \tilde{A})
$$

where $d(A, \tilde{A})$ denotes the distance between set $\mathrm{A}$ and its nearest ordinary set $\tilde{A}$ and $\mathrm{n}$ is the number of elements in $A$. An ordinary set $\tilde{A}$ nearest to the fuzzy set $A$ is defined as

$$
\mu_{\tilde{A}}\left(x_{i}\right)= \begin{cases}0 & \text { if } \mu_{A}\left(x_{i}\right) \leq 0.5 \\ 1 & \text { if } \mu_{A}\left(x_{i}\right)>0.5\end{cases}
$$

Based on the value of $\mathrm{k}$, there are two types of index, i.e. linear and quadratic. Linear $I o F$ may be written as Equation (6).

$$
\gamma_{A}=\frac{2}{n} \sum_{g=0}^{n-1} \min \left(\mu_{A}\left(a_{i}\right), 1-\mu_{\tilde{A}}\left(a_{i}\right)\right)
$$

Meanwhile, Tizhoosh [7] defines a linear $I o F, \gamma_{X}(g)$ for a $\mathrm{M} \times \mathrm{N}$ image subset $\mathrm{A} \subseteq \mathrm{X}$ with $\mathrm{L}$ graylevel $\mathrm{g} \in$ $[0, \mathrm{~L}-1]$, the histogram $h(\mathrm{~g})$, and the membership function $\mu_{X}(\mathrm{~g})$ as follows.

$$
\gamma_{X}(g)=\frac{2}{M N} \sum_{g=0}^{L-1} h(g) \times \min \left(\mu_{A}(g), 1-\mu_{A}(g)\right)
$$

\subsection{Thresholding Algorithm using Fuzzy Similarity Measure}

Lopes et al [2] and Pratamasunu etal. [9] using fuzzy similarity measure to image segmentation. To calculate it at each histogram level, it is necessary to specify the fuzzy region first. At Lopes et al [2], fuzzy region boundary search using statistical parameters, $P_{1}$ and $P_{2}$. If $B_{\text {seed }}$ is the initial region of black/dark pixels, $W_{\text {seed }}$ is the initial region of white/bright pixels, and $h\left(x_{\mathrm{i}}\right)$ shows the number of occurrences at graylevel, then $P_{1}$ has gotten from minimal search a function of the number of pixels in the graylevel intervals $[0,127]$ dan $[128,255]$ with Equation (5). 


$$
\operatorname{MinPix}_{\text {Bseed }\left(\mathrm{W}_{\text {seed }}\right)}=P_{1} \sum_{i=0(128)}^{127(255)} h\left(x_{i}\right)
$$

The value of $\mathrm{P}_{1} \in[0,1]$. Meanwhile, $\mathrm{P}_{2}$ is obtained from Equation (6).

$$
P_{\mathrm{MIN}}=P_{2} M N
$$

If the number of pixels including to the graylevel intervals $[0,127]$ or $[128,255]$ is smaller than a value $P_{\text {MIN }}$, so the image histogram must be equalized. Based on an experiment using 30 images obtained the value of P1 and $\mathrm{P} 2$ is $39,64 \%$ and $20 \%$, respectively. At Pratamasunu et al [9], fuzzy region boundary is defined based on the fuzzy region centre. For the fuzzy region centre $V_{C}$ and number of pixels on graylevel $g, h(g)$, boundaries of initial seed for dark and bright region, $V_{B}$, and $V_{W}$, are defined as

$$
\mathrm{V}_{B}=\frac{\sum_{g=0}^{V_{C}} g \cdot h(g)}{\sum_{g=0}^{V_{C}} h(g)} \text { and } V_{W}=\frac{\sum_{g=V_{C}}^{L=1} g \cdot h(g)}{\sum_{g=V_{C}+1}^{L=1} h(g)}
$$

The fuzzy region boundary search process is done along graylevels. The fuzzy regions selected are a region with the highest $I o F$. The general algorithm of Pratamasunu etal. [9] proposed method as follows:

Step 1. Compute the image histogram

Step 2. Move $\mu_{B}$ and $\mu_{W}$ throughout graylevels

Step 3. Compute $I o F, \gamma$, in each graylevel

Step 4. Determine the centre of fuzzy region, $V_{\mathrm{C}}=\arg \max (\gamma)$

Step 5. Compute the boundaries of seed subsets, $V_{B}$ and $V_{W}$

Step 6. For all graylevels $f_{i}$ in fuzzy region calculate $\zeta\left(B \cup\left\{f_{i}\right\}\right)$ and $\zeta\left(W \cup\left\{f_{i}\right\}\right)$

Step 7. Threshold the image with $\mathrm{T}=\arg \max \left(\zeta\left(B \cup\left\{f_{i}\right\}\right), \zeta\left(W \cup\left\{f_{i}\right\}\right)\right)$

where $\zeta\left(S \cup\left\{f_{i}\right\}\right)=\frac{\sum_{g=1}^{n}\left(g-M\left(S \cup\left\{f_{i}\right\}\right)\right)^{2}}{\sum_{g=1}^{n} h(g)}$ and $M(A)=\sum_{g=1}^{n} h(g) \times g \times\left|\left(\mu_{A}(g)-\mu_{A^{*}}(g)\right)\right|$

\subsection{Adaptive thresholding by Anitha et al.}

Anitha et al [12] performed a refinement segmentation in mass detection in mammograms based on Histogram Peak Analysis (HPA) using adaptive window based thresholding approach. The threshold is computed for each pixel in the region of interest, to recognize if the pixel is suspicious (1) or normal (0) by setting a small window, W, sized $15 \times 15$. This window-based adaptive thresholding is based on the intensity contrast that marks the intensity divergence between mass and background. To increase the selection of thresholds for each pixel, $I(x, y)$, the algorithm to refine the result segmentation of a pixel, $B_{\text {fine }}(x, y)$, is presented as follows.

$$
\text { If } \begin{aligned}
W_{\text {diff }}(x, y) & \leq T_{C} \text { Then } \\
B_{\text {fine }}(x, y) & =\left\{\begin{array}{lc}
1 & \left.I(x, y) \geq \delta \times W_{\text {diff }}(x, y)+I_{\text {mean }}\right) \\
0 & \text { else }
\end{array}\right.
\end{aligned}
$$

Else

$$
B_{\text {fine }}(x, y)=\left\{\begin{array}{cc}
1 & \left.I(x, y) \geq W_{\text {mean }}(x, y)\right) \\
0 & \text { else }
\end{array}\right.
$$

where $W_{\text {diff }}(x, y)=W_{\max }(x, y)-W_{\min }(x, y)$ and $T_{\mathrm{C}}=\left(I_{\max }+\right.$ mean of all pixel greater than $\left.I_{\max }\right) / 2$. $\delta$ is a bias threshold coefficient. It takes values in the range of 0 to 1 . The value is set empirically as $\delta=0.5 . I_{\operatorname{mean}}$ and $I_{\max }$ 
are the mean and maximum intensity of the image. $\mathrm{W}_{\max }(),. \mathrm{W}_{\min }($.$) , and \mathrm{W}_{\text {mean }}($.$) are the maximum,$ minimum, and mean intensity values in the small window, respectively. The computed threshold for each pixel makes a refinement segmentation that gives an exact segmentation of the suspicious lesion.

\section{RESEARCH METHOD}

\subsection{Material}

Data for this research (original image and ground truth) are provided from grayscale images from Lopes et al [2], and Pratamasunu et al [9]. Moreover, experiment data are images of malaria parasite candidate with grayscale JPG format. The data are obtained from Malaria slide of Eijkman Institute for Molecular Biology. The extraction of the thickblood smear is based on hierarchical classification based on the morphological characteristics of connected components. The ground truths were built based on Malaria Diagnosis Guideline by expert instruction.

\subsection{Proposed Method}

The purpose of this paper is to perform binary segmentation on an image that divides the image into foreground and background areas. This type segmentation needs well-defined image, i.e. having bimodal (or nearly) histograms. Very irregular histogram is defined by Tobias and Siera [8] as the cases where the image histogram may only have local minimum, or even not have any minimum at all. Example of very irregular histogram is shown as Figure 1c and 1d. The proposed method consists of two main parts, i.e. perform the initial thresholding on the image using $I o F$ and refinement pixels using Adaptive thresholding.

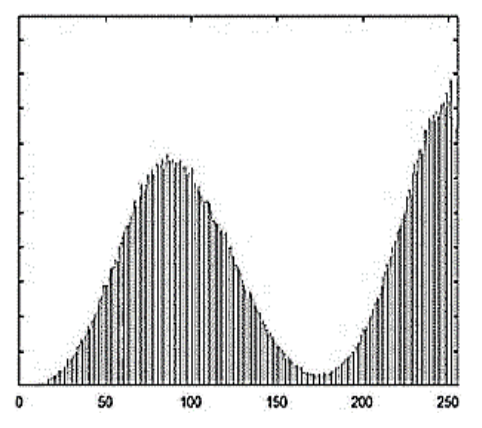

a

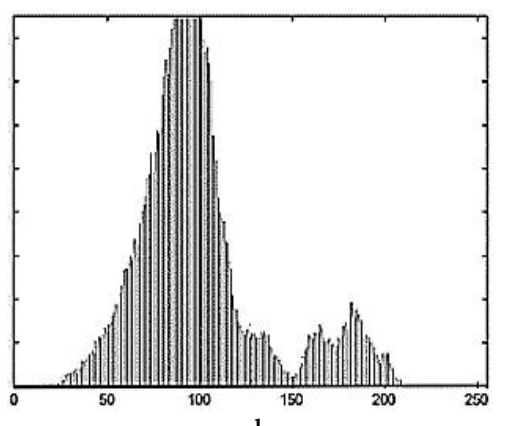

b

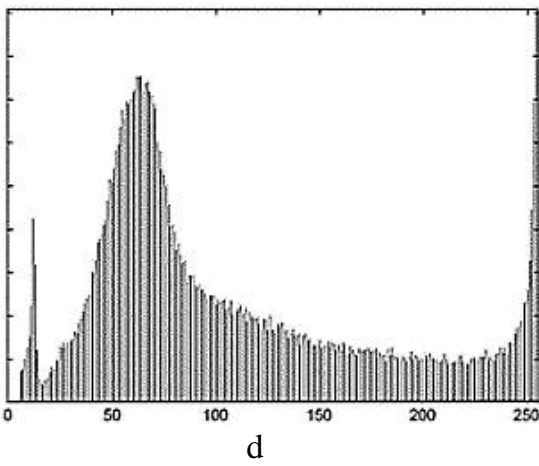

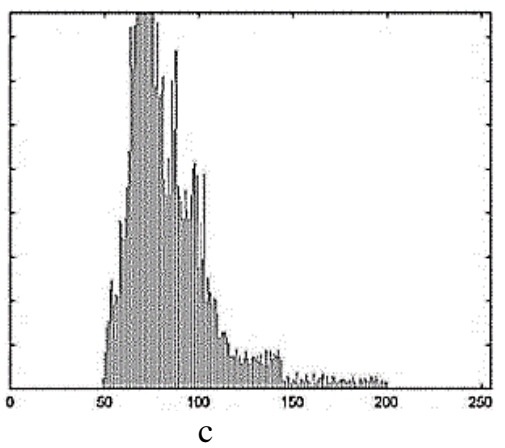

$\mathrm{C}$

Figure 1. Modal of histogram image: a. bimodal, b. nearly bimodal, c. unimodal, d. Multimodal

\subsubsection{Measurement of Index of Fuzziness}

Two memberships are built to calculate the $I o F$, i.e. dark region $(B)$ and bright region $(W)$ as shown in Figure 2a. The S and Z functions are used to model dark and bright region, respectively [11]. Formulation of both functions in (9) and (10). 


$$
\mu_{A S}(x)=S(x ; a, b, c)= \begin{cases}0, & x<a \\ 2\left\{\frac{x-a}{c-a}\right\}^{2}, & a \leq x \leq b \\ 1-2\left\{\frac{x-a}{c-a}\right\}^{2}, & b<x \leq c \\ 1, & x>c\end{cases}
$$

with initial parameters a and $\mathrm{c}$ are taken from the lowest and highest level of the image histogram, while $\mathrm{b}$ is defined as $b=(a+c) / 2$.

$$
\mu_{A Z}(x)=Z(x ; a, b, c)=1-S(x ; a, b, c)
$$

In this work, $I o F$ is calculated using Equation (4) for each fuzzy subset region by utilizing the appropriate fuzzy membership. The formulation of the $I o F$ for each graylevel of dark and light areas respectively such as Equations (11) and (12).

$$
\gamma_{B}=\frac{2}{N} \sum_{g=0}^{L-1}\left[h(g) \times \mu_{B}(g)\right]
$$

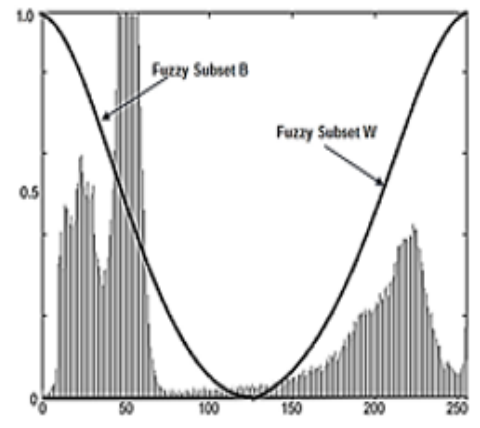

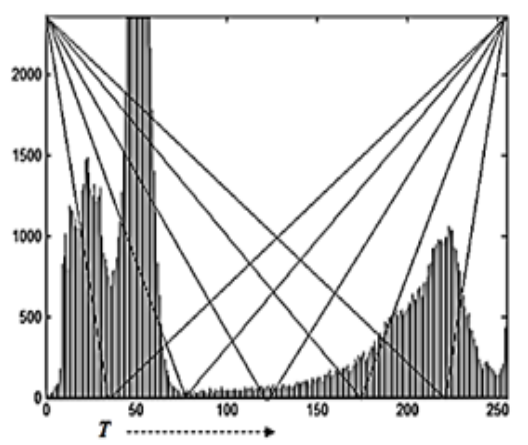

b.

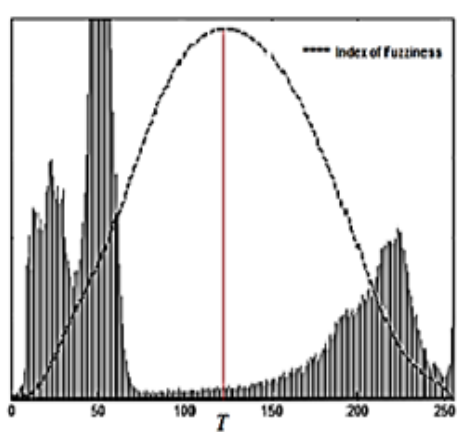

Figure 2. Threshold determination process: a. Membership function of dark and bright areas,

b. Calculation process of Index of Fuzziness each graylevel, c. Finding the maximum Index of Fuzziness

$$
\gamma_{W}=\frac{2}{N} \sum_{g=0}^{L-1}\left[h(g) \times \mu_{W}(g)\right]
$$

Multiplication of both indexes determines the total index for each graylevel.

$$
\begin{aligned}
& \gamma_{T}=\gamma_{B} \cdot \gamma_{W} \\
& \gamma_{T}=\frac{4}{N^{2}} \sum_{g=0}^{L-1}\left[h(g) \times \mu_{B}(g)\right] \sum_{g=0}^{L-1}\left[h(g) \times \mu_{W}(g)\right]
\end{aligned}
$$

The process of calculating $I o F$ from each graylevel position is performed by moving membership functions ( $\mu_{\mathrm{B}}$ and $\mu_{\mathrm{W}}$ ) throughout graylevels that are restrained by a point $\mathrm{T}$ as shown in Figure 2b. The threshold can be determined by graylevel with the maximum $I o F$ as shown in Figure 2c. Coarse segmented image, $B$, obtained by performing this threshold on the image. Figure 3 is an example of image segmentation result using $I o F$ based threshold. 


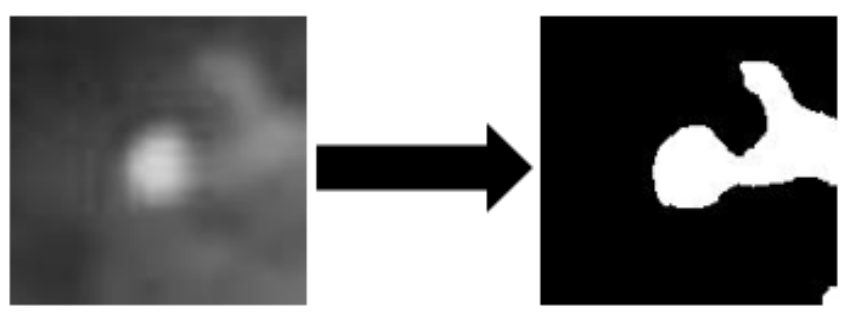

Figure 3. Example of result segmentation

\subsubsection{Refinement Pixel}

Coarse segmentation result from the previous process is compared with ground truth. There are two types of misclassification, i.e. the background is predicted as the object called false positive (FP) and the object is predicted as background called false negative (FN). From the data in Figure 4, the threshold obtained using IoF is 109. The number of FP and FN are 93 and 185, respectively. The distribution of number of misclassification pixel and the corresponding number of true classified pixel is presented in Figure 4.

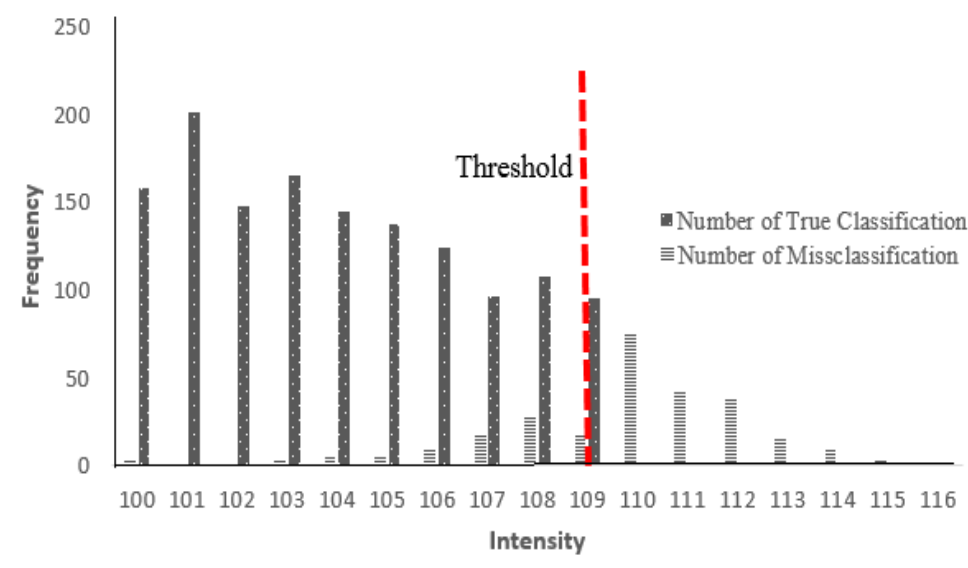

Figure 4. Distribution of number of misclassification pixel

Misclassification occurs in pixels with intensities around the threshold [13]. It happens because pixels at the same intensity can belong to foreground or background depending on the relation of a pixel to the surrounding pixels. Such cases often occur in images that have different illumination, noise, or the condition of some objects have an intensity similar to the background. Therefore, a refinement on these pixels is needed. The fixed threshold is rather unlikely in this situation, a local threshold is more appropriately used to suit the varying lighting conditions and changing background [14]-[18].

Refinement of the segmentation results for each pixel, $B_{\text {fine }}(x, y)$, with the intensity around the threshold is based on local contrast methods that utilize the statistical properties of previous segmentation results. The refinement operation is performed on some neighbourhood ( $w \times w$ window). The comparison is made between the average pixel intensity in the window of an original image $\left(W_{\text {mean }}\right)$ and the average pixel intensity in the foreground region of the initial segmentation results $\left(R O I_{\text {mean }}\right)$ as in Figure 5. The refinement algorithm for the initial pixel segmentation results, $B_{\text {fine }}(x, y)$, as in (16).

$$
B_{\text {fine }}(x, y)=\left\{\begin{array}{cc}
1 & W_{\text {mean }}(x, y) \geq R O I_{\text {mean }} \\
0 & \text { else }
\end{array}\right.
$$

Moreover, the refinement algorithm may also use adaptive thresholding (8) proposed by Anitha et al [13] based on $h(T)$. 

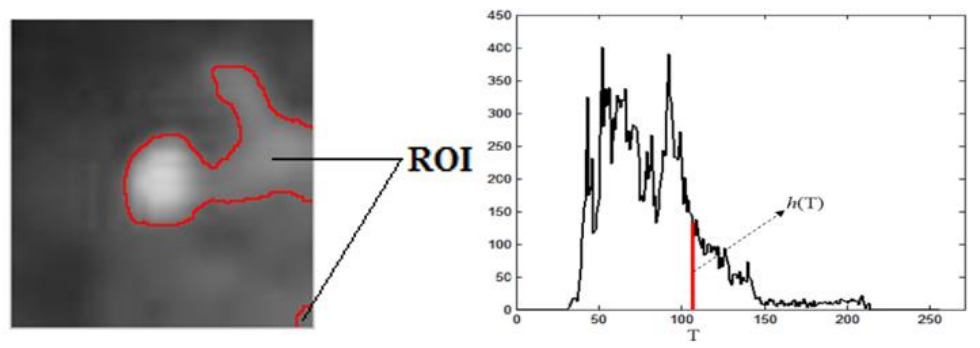

Figure 5. ROI and the histogram

\subsubsection{Proposed Thresholding Algorithm}

The general algorithm of the proposed method describes as follows.

Step 1. Compute the image histogram

Step 2. Move $\mu_{B}$ and $\mu_{W}$ throughout graylevels

Step 3. Compute $I o F, \gamma$, in each graylevel

Step 4. Find $T=\arg \max (\gamma)$

Step 5. Threshold the image with $T$

Step 6. Find number of pixel at $T, h(T)$

Step 7. For all pixels that have intensities on interval $V_{\mathrm{C}} \pm \alpha$ do refinement to the segmentation result in Step 5. If $h(\mathrm{~T})$ is low, then use Equation (8), else use Equation (16).

\section{RESULTS AND DISCUSSION}

To illustrate the performance, used experiment data are from related research papers, i.e. six images from Lopez et al [2] and Pratamasunu et al [9] and five noisy images from Pratamasunu et al [9]. Moreover, the experiment also uses ten parasite candidate images that have been extracted from malaria slide of some thickblood smear. Comparison method uses Otsu and thresholding method based fuzzy similarity measure. Some given parameters are as follows. The size of window is $3 \times 3, \alpha=4$, and the low limit $h(t)$ is 100 . The performance measure of segmentation uses Accuracy Segmentation $(A c c)$ that measure the ratio of pixels that truly classified. Acc can be expressed as

$$
A c c=\frac{\left(\left|B_{o} \cap B_{T}\right|+\left|F_{o} \cap F_{T}\right|\right)}{\left|B_{o}\right|+\left|F_{o}\right|} \times 100 \%
$$

where $\mathrm{F}_{\mathrm{o}}, \mathrm{B}_{\mathrm{o}}, \mathrm{F}_{\mathrm{T}}$ and $\mathrm{B}_{\mathrm{T}}$ are the foreground and background pixels in the ground truth image and the segmentation result, respectively. The range of $A c c$ value is $[0,100]$. If the Acc value is 100 then the segmentation result is same with the ground truth image. Six testing images from Lopez et al [2] and Pratamasunu et al. [9] are used in the first experiment. Segmentation is performed using segmentation method proposed by Pratamasunu etal. (Fuzzy Similarity Measure, FSM), proposed method (Fuzzy Indexes and Automatic Thresholding, FIAT), and Otsu Thresholding (Otsu). The result is shown in Figure 6. The histogram of an original image is presented as Figure 7.

Visually, the result of segmentation using FSM method is almost the same as the proposed method. Otsu thresholding gets slightly worse results than both. Quantitatively, the segmentation accuracy of such methods is presented in Table 1. Segmentation accuracy using IoF (Fuzzy Indexes, FI) and segmentation method that proposed by Lopes etal. without and with histogram equalization (EM1 and EM2) is added in that table.

There are several analyzes related to the use of compared segmentation method based on Table 1. Firstly, the use of FI can provide satisfactory accuracy results. It proven from greater than $90 \%$ accuracy for all sample images. Secondly, the use of fuzzy similarity measure-based method, EM2 and FSM, have improved accuracy and obtained the highest results on images that they have deep valley histograms and clear objects than the background (Gearwheel, Potatoes, and Stone image). However, the accuracy decrease compared to segmentation results by FI method on images that have a very irregular histogram and low-contrast. Finaly, the use of the proposed method, FIAT, can improve overall accuracy compared to FI 
and achieves the highest accuracy on images that have very irregular histogram and low-contrast between object and background.

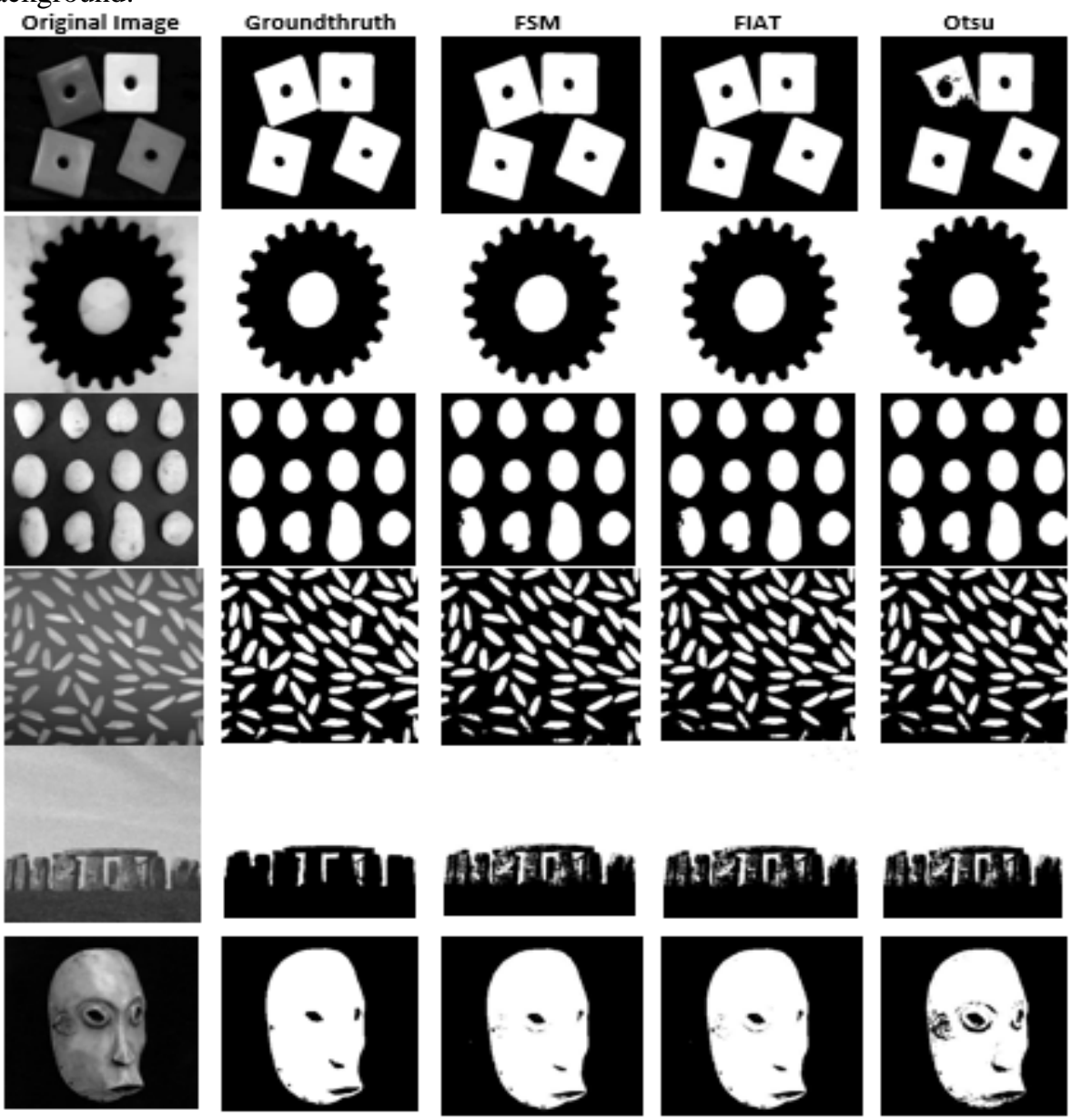

Figure 6. Result segmentation on Blocks, Gearwheel, Potatoes, Rice, Stone, and Zimba images, respectively

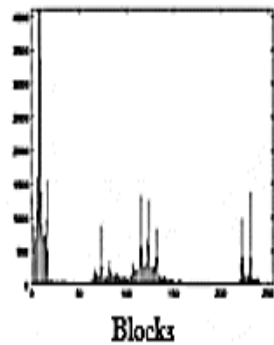

Blocks

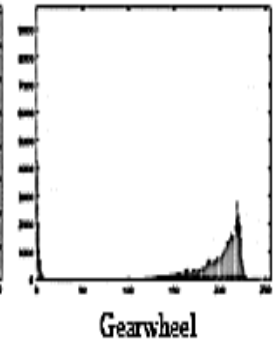

Gearnheel

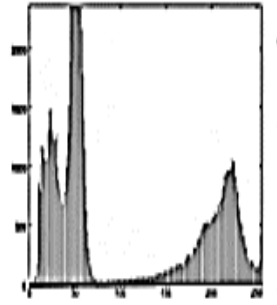

Potatoes

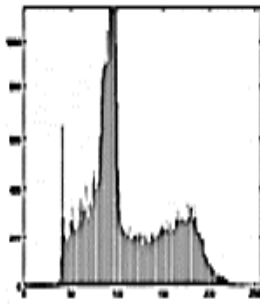

Rice

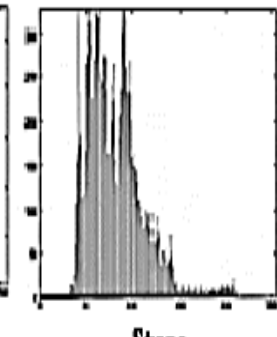

Stone

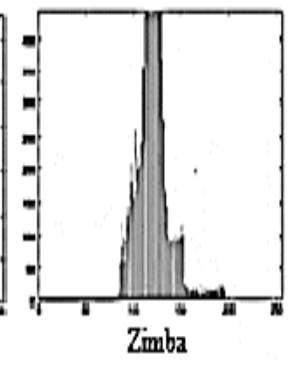

Figure 7. Histogram of first group images

Second experiment uses synthetic reference image from Pratamasunu et al [10]. Four images else are composed with different graylevel intervals from this reference image. These images are obtained by adding increasing Gaussian noises as shown in Figure 8. It can be seen that increased noise causes a decrease in the steepness of the valley formed on the image histogram. Increasing noise decreases the separation between object and background.

The segmentation performance results of several methods to this data are shown in Table 2. Segmentation accuracy with FSM is outstanding when the image histogram has a clear separation between objects and backgrounds marked by deep valley on the histogram as shown in Figure 8d and 8e. Meanwhile, the proposed method is outstanding in the opposite condition. Based on the average accuracy of the five images, the proposed method is outstanding than FSM and Otsu. 


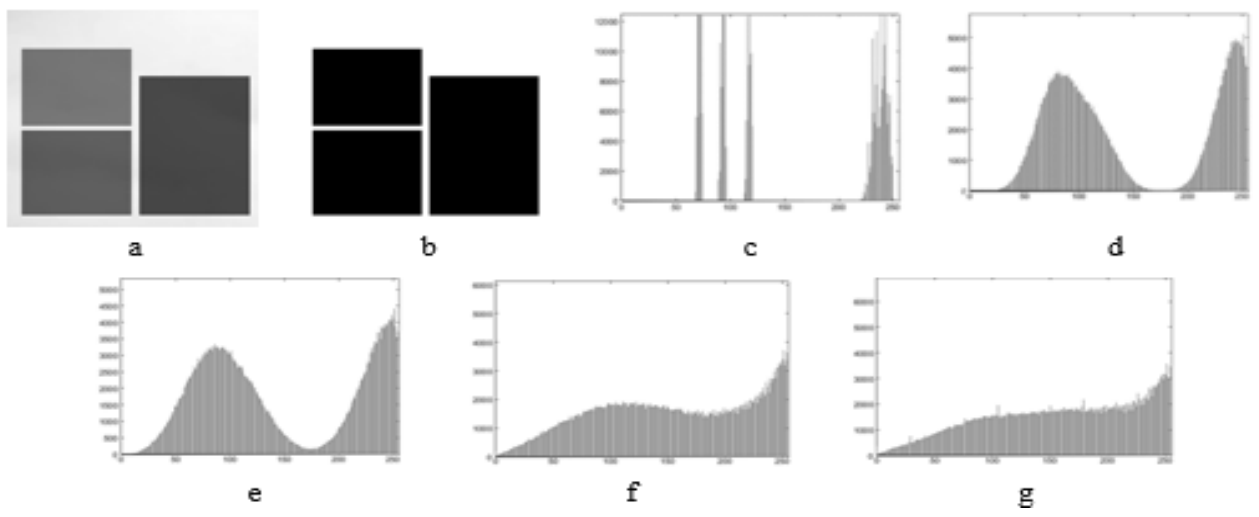

Figure 8. Synthetic reference image: a. The original image, b. Ground truth, c. Histogram of the original image, $\mathrm{d}-\mathrm{g}$. Histogram of original image that give Gaussian Distribution noises $g(0.01,0.01), g(0.05,0.05)$, $g(0.10,0.10)$, and $g(0.15,0.15)$, respectively

Table 2. Segmentation Accuracy from Second Group Sample

\begin{tabular}{ccccc}
\hline \multirow{2}{*}{ No } & \multicolumn{1}{c}{ Image } & \multicolumn{3}{c}{ Acc $(\%)$} \\
\cline { 3 - 5 } & & FSM & FIAT & Otsu \\
\hline 1 & Original & 100 & 100 & 100 \\
2 & Original+ $g(0.01,0.01)$ & $\mathbf{9 9 . 9 9}$ & 99.96 & 99.89 \\
3 & Original+ $g(0.05,0.05)$ & $\mathbf{9 9 . 6 4}$ & 99.56 & 99.04 \\
4 & Original+ $g(0.10,0.10)$ & 88.49 & $\mathbf{9 0 . 2 5}$ & 82.97 \\
5 & Original+ $g(0.15,0.15)$ & 84.91 & $\mathbf{8 5 . 0 5}$ & 76.46 \\
\hline \multicolumn{2}{c}{ Average } & 94.61 & $\mathbf{9 4 . 9 6}$ & 91.67 \\
\hline
\end{tabular}

Figure 9 shows graphs of the $I o F$ for each graylevel from the five images in the second experiment. It can be seen that the addition of noise to the original image causes the $I o F$ graph change, but the graylevel position with maximum value does not have much effect since it does not move much from the position without noise. It shows that the use of $I o F$ to determine the threshold is robust to noise. Refinement pixelpixel on segmentation results around the threshold of the noisy image to be the right choice to improve the accuracy of segmentation results.

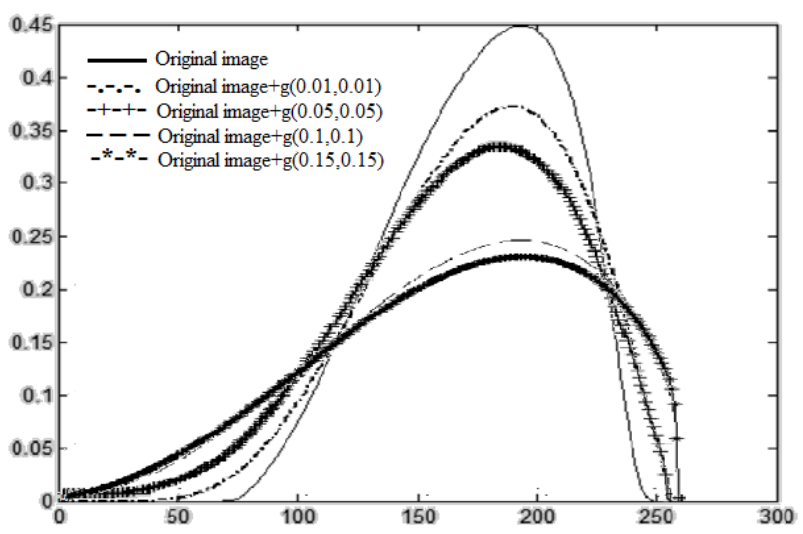

Figure 9. Index of Fuzziness Graph from images of the second experiment

In the third experiment, we used grayscale images of parasite candidate from thickblood smear as shown Figure 10. A malaria parasite is characterized by a nucleus with attached cytoplasm [19]. The presence of the cytoplasm is important for distinguishing a parasite's nucleus from an object whose shape and size resemble parasite's nucleus [20], [21]. The size of each file is $140 \times 140$ pixels that derived from 16 times 
bicubic magnification from an extraction of malaria parasite candidate $35 \times 35$ pixels. Most of these images have a very irregular histogram and low-contrast. This can be seen from the image histograms in Figure $11 \mathrm{~b}$.

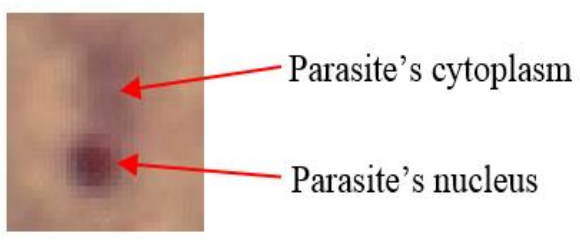

a

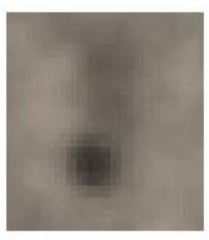

b

Figure 10. Malaria parasite candidate: a. RGB images, b. Grayscale image

Most of the segmentation results with the FSM method are under segmentation as shown in Figure 11d, so the resulting object is smaller than the ground truth. Even some images lose cytoplasm (3rd, 6th, and 8th candidate). This condition is opposite with result segmentation using Otsu method that over-segmentation as shown in Figure 11f. Results segmentation of the proposed method as shown in Figure 11e are more similar to the groundtruth than two other methods for almost any image. Different results are shown on the 5th and 6th candidate images. Both images have a bimodal (or nearly) histogram with a deep valley between them. Accuracy segmentation 5th parasite candidate image using FSM method is higher than others. It has a clear distinction between objects and background. Meanwhile, in the 6th candidate image, the object is clearly visible although the contrast is not too high, the background conditions are homogeneous. For this image, the Otsu method produces segmentation results that are more similar to ground truth.

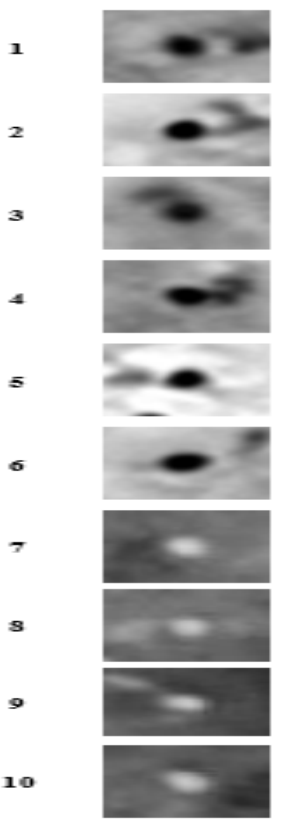

a

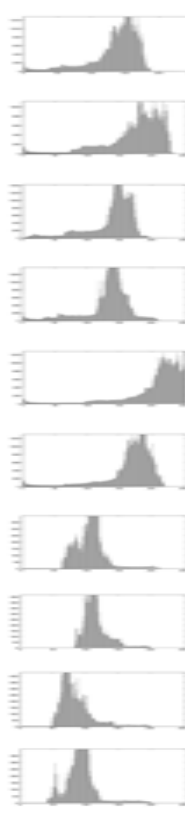

b

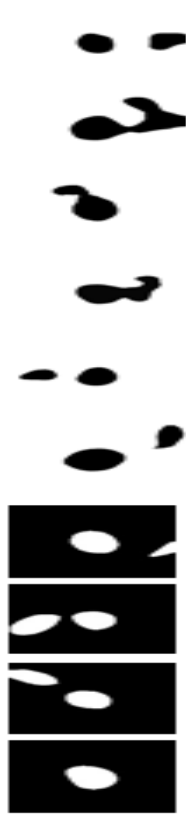

C

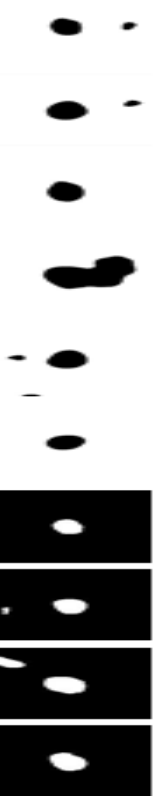

d

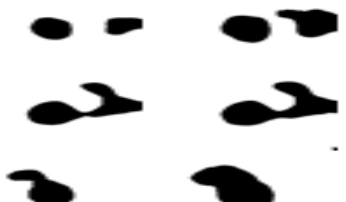

2
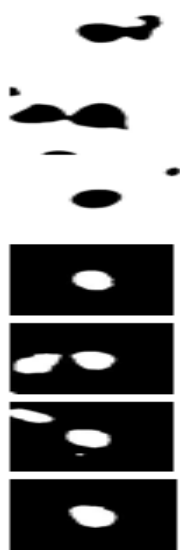

e
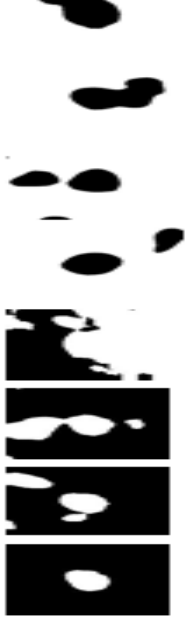

f

Figure 11. Result segmentation of parasite candidate: a. The original image, b. Histogram image, c. Groundtruth, d-f. Result segmentation of FSM, proposed method, and Otsu, respectively

Beside accuracy segmentation, we define performance that measures the distance between the ideal threshold $\left(\mathrm{T}_{\text {Ideal }}\right)$, i.e. the optimal threshold performed manually based on visual inspection and threshold generated by the segmentation method $\left(\mathrm{T}_{\mathrm{Comp}}\right)$ called Estimation Error $(\mathrm{EE})$. Smaller the value of EE means the better the segmentation results. The formulation of $\mathrm{EE}$ as defined in Equation (18). 


$$
E E=\left|\frac{\mathrm{T}_{\text {Ideal }}-\mathrm{T}_{\text {Comp }}}{255}\right| \times 100 \%
$$

Performance of segmentation methods on the parasite candidates quantitatively is shown in Table 3. The results show that the proposed method can produce the closest threshold to the ideal threshold. Moreover, it has the highest accuracy and lowest estimation errors in most of the images. By results of the previous visual analysis, FSM excels quantitatively at the fifth candidate, while Otsu excels at the 6th candidate. This result shows that the proposed method is properly implemented in parasite candidate images.

Table 3. Performance of segmentation methods from Third Group Sample

\begin{tabular}{|c|c|c|c|c|c|c|c|c|c|c|}
\hline \multirow{2}{*}{ Image } & \multicolumn{4}{|c|}{ Threshold } & \multicolumn{3}{|c|}{ Estimation Error (\%) } & \multicolumn{3}{|c|}{$\operatorname{Acc}(\%)$} \\
\hline & Ideal & FSM & FIAT & Otsu & FSM & FIAT & Otsu & FSM & FIAT & Otsu \\
\hline Candidate 1 & 75 & 47 & 82 & 108 & 10.98 & 2.75 & 12.94 & 95.68 & 97.92 & 91.07 \\
\hline Candidate 2 & 130 & 81 & 124 & 139 & 19.22 & 2.35 & 3.53 & 88.66 & 97.63 & 97.08 \\
\hline Candidate 3 & 80 & 63 & 87 & 112 & 6.67 & 2.75 & 12.55 & 95.49 & 98.55 & 91.75 \\
\hline Candidate 4 & 70 & 108 & 66 & 98 & 14.9 & 1.57 & 10.98 & 84.1 & 89.73 & 85.87 \\
\hline Candidate 5 & 125 & 106 & 171 & 159 & 7.45 & 18.04 & 13.33 & 96.87 & 92.32 & 94.08 \\
\hline Candidate 6 & 120 & 39 & 75 & 122 & 31.76 & 17.65 & 0.78 & 91.94 & 94.14 & 98.05 \\
\hline Candidate 7 & 130 & 171 & 145 & 104 & 16.08 & 5.88 & 10.20 & 91.31 & 91.33 & 45.7 \\
\hline Candidate 8 & 137 & 156 & 141 & 129 & 7.45 & 1.57 & 3.14 & 88.73 & 93.25 & 89.41 \\
\hline Candidate 9 & 117 & 123 & 118 & 104 & 2.35 & 0.39 & 5.10 & 96.94 & 98.19 & 95.63 \\
\hline Candidate 10 & 125 & 147 & 126 & 124 & 8.63 & 0.39 & 0.39 & 92.41 & 92.52 & 92.51 \\
\hline \multicolumn{5}{|c|}{ Average } & 12.55 & 5.33 & 7.29 & 92.21 & 94.56 & 88.12 \\
\hline
\end{tabular}

Based on the experimental results of the three datasets above, it can be concluded that the proposed method is suitable for use on binary segmentation of images that have very irregular histogram and low-contrast. The use of the method on multi thresholding cases will be further research.

\section{CONCLUSION}

A new strategy to overcome segmentation problem on images that have a very irregular histogram and not significant contrast is presented in this paper. The strategy is incorporating $I o F$ and adaptive thresholding. Initial threshold is obtained by finding graylevel with the maximum IoF. Furthermore, segmentation is performed on the image with the initial threshold so that the coarse segmented are obtained. Better segmentation results are obtained from implemented local thresholding based on an adaptive window. Experimental results show that the proposed segmentation method gives improved accuracy than any other methods. Meanwhile, fuzzy similarity measure based segmentation method is more suitable to be used on images that have a deep valley on the histogram and significant contrast. In the future, the criterion or size of the irregularity of the histogram needs to be mathematically defined so that the exact method can be determined for different image conditions.

\section{ACKNOWLEDGEMENTS}

This research was supported by Ministry of Research, Technology and Higher Education of the Republic of Indonesia under Grant PDD 2017 with contract number is 873/UN27.21/PP/2017. We are grateful to Mr Pratamasunu and Eijkman Institute for Molecular Biology Indonesia for preparing the materials for this research and giving access to the datasets. We are also grateful to Mr Pratamasunu for the supports of comparison program for this research.

\section{REFERENCES}

[1] Weszka JS. A survey of threshold selection techniques. Computer Graphics and Image Processing. 1978; 7(2): 259-265.

[2] Lopes NV, Mogadouro PA, Bustince H, Melo-pinto P. Automatic Histogram Threshold Using Fuzzy Measures. IEEE Transactions On Image Processing. 2010; 19(1): 199-204. 
[3] Sezgin M, Sankur B. Survey over image thresholding techniques and quantitative performance evaluation. Journal of Electronic Imaging. 2004; 13(1): 146-165.

[4] Otsu N. A threshold selection method from gray-level histograms. IEEE Transaction on System, Man, and Cybernetics. 1979; 9: 62-66.

[5] Arifin AZ, Asano A. Image segmentation by histogram thresholding using hierarchical cluster analysis. Pattern Recognition Letters. 2006.

[6] Huang LK, Wang MJJ. Image Thresholding by Minimizing The Measure of Fuzziness. Pattern Recognition. 1995; 28(1): 41-51.

[7] Tizhoosh HR. Image thresholding using type II fuzzy sets. Pattern Recognition. 2005; 38: 2363-2372.

[8] Tobias OJ, Seara R. Image Segmentation by Histogram Thresholding Using Fuzzy Sets. IEEE Transactions On Image Processing. 2000; 11(12): 1457-1465.

[9] Pratamasunu GQO, Arifin AZ, Navastara DA, Wijaya AY, Khotimah WN. Image Thresholding Based on Index of Fuzziness and Fuzzy Similarity Measure. IEEE 8th International Workshop on Computational Intelligence and Applications. Hiroshima, Japan. 2015.

[10] Pal SK, King RA, Hashim AA. Automatic grey level thresholding through index of fuzziness and entropy. Pattern Recognition Letters. 1983; 1( 3): 141-146.

[11] Bustince H, Herrera F, and Montero J. Fuzzy Sets and Their Extensions: Representation, Aggregation and Models. Springer, 2008.

[12] Anitha J, Peter JD, and Alex SI. A dual stage adaptive thresholding (DuSAT) for automatic mass detection in mammograms. Computer Methods and Programs in Biomedicine. 2017; 138: 93-104.

[13] Jamal I, Akram MU, Tariq A. Retinal Image Preprocessing: Background and Noise Segmentation. TELKOMNIKA. 2012; 10( 3): 537-544.

[14] Zhang W, Dou J. A Fast Thresholding Technique in Image Binarization for Embedded System. TELKOMNIKA Indonesian Journal of Electrical Engineering. 2014; 12(1): 592-598.

[15] Singh OI, James O, Sinam T, Singh TR. Local Contrast and Mean based Thresholding Technique in Image Binarization. International Journal of Computer Applications. 2012; 51(6): 5-10.

[16] Hore S, et al. An Integrated Interactive Technique for Image Segmentation using Stack based Seeded Region Growing and Thresholding. International Journal of Electrical and Computer Engineering. 2016; 6(6): 27732780.

[17] Yazid H, Arof H. Gradient based adaptive thresholding. Journal of Visual Communication and Image Representation. 2013; 24(7): 926-936.

[18] Singh TR, Roy S, Singh OI, Sinam T, Singh KM. A New Local Adaptive Thresholding Technique in Binarization. International Journal of Computer Science Issues. 2011; 8(6): 271-277.

[19] WHO, Basic Malaria Microscopy, 2nd ed. Switzerland: WHO Press, 2010.

[20] Salamah U, et al. Enhancement of Low Quality Thickblood Smear Microscopic Images of Malaria Patients using Contrast and Edge Corrections. International Conference on Knowledge Creation and Intelligent Computing (KCIC). Menado, Indonesia. 2016; 219-225.

[21] Abidin SR, Salamah U, Nugroho AS. Segmentation of Malaria Parasite Candidates from Thick Blood Smear Microphotographs Image Using Active Contour Without Edge. International Conference on Biomedical Engineering (IBIOMED). Yogyakarta, Indonesia. 2016; 78-83.

\section{BIBLIOGRAPHY OF AUTHORS}

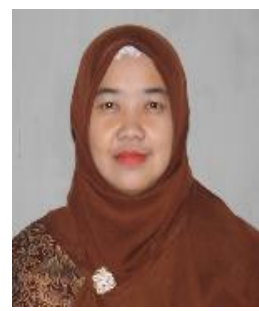

Umi Salamah received her Master degree in 2002 and she is currently a Ph.D. student in the Department of Computer Science at the Institut Teknologi Sepuluh Nopember Surabaya, Indonesia. Her research interests include image processing and pattern recognition in computer aided diagnosis for malaria from thick blood smears microphotograph

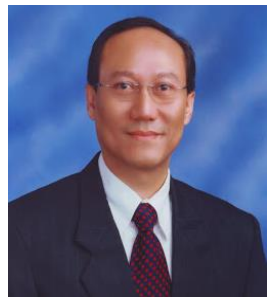

Riyanarto Sarno received his M.Sc and Ph.D in Computer Science from the University of Brunswick Canada in 1988 and 1992. His research includes Internet of Things, Enterprise Computing, Information Management, Intelligent Systems and Smart Grids 


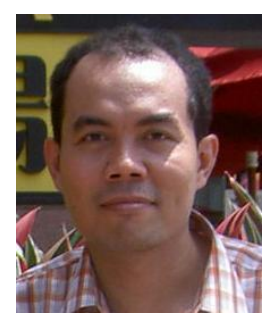

Agus Zainal Arifin received his Master degree in Computer Science University of Indonesia in 2001 and Dr. degree in Information Engineering from Hiroshima University, Japan in 2007. His research interests included Pattern Recognition, Image Processing, Computer vision, and Computational intelligent

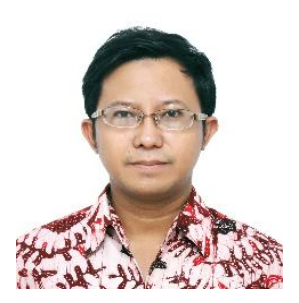

Anto Satriyo Nugroho received his M.Eng. and Dr.Eng. degrees in Electrical and Computer Engineering from Nagoya Institute of Technology of Japan, in 2000 and 2003 respectively. His research interests included Pattern Recognition \& Image processing with applied field of interests on Biometrics \& Computer aided diagnosis for Malaria status identification from blood smears microphotograph. Currently, he is the President of Indonesian Association for Pattern Recognition (INAPR), elected in 2017

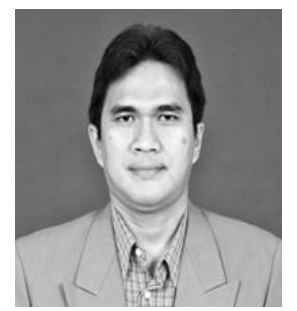

Ismail Ekoprayitno Rozi received his Master degree in Bioengineering from Nagaoka University of Technology in 1995. Now he is working as senior researcher in bioinformatics unit and IT officer of Eijkman Institute for Molecular Biology. He is interested in computer aided diagnosis for malaria from blood smears

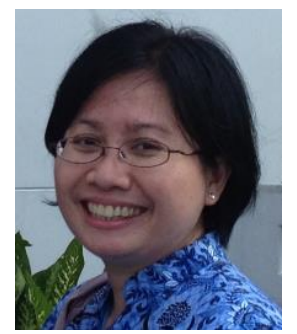

Puji Budi Setia Asih earned her doctoral degree in 2010 from University of Indonesia. Now she is currently the head of Malaria and vector resistance Department at the Eijkman Institute for Molecular Biology. She has involved in several research projects such as molecular mechanisms underlying Plasmodium resistance to anti malarial drugs and anti malarial drug discovery through medicinal plants. 